\title{
On the Representation of Sensor Faults in Fault Detection Filters*
}

\author{
JAEHONG PARK, $\dagger$ GIORGIO RIZZONI $\ddagger$ and WILLIAM B. RIBBENS $\dagger$
}

Key Words-Failure detection; observers; sensor failures.

\begin{abstract}
This paper presents an extension of the wellknown Beard-Jones detection filter that permits isolation of sensor faults in a dynamic system to a fixed direction in output space. The method is based on augmenting the system equations by an auxiliary state to represent the dynamic behavior of the sensor fault, and in effect converts the sensor fault into the same form as an actuator fault. The only condition required is observability of the original system.
\end{abstract}

\section{Introduction}

THIS PAPER IS concerned with the formulation of a method for the detection and isolation of sensor faults in dynamic systems. The proposed method is an extension of the well-known Beard-Jones detection filter. In such fault detection and isolation (FDI) methods, a model of the system is used to predict the plant outputs. The residual vector (i.e. the difference between measured and calculated outputs) is then examined. In the ideal case (zero noise and perfect reference model), the residual vector is zero as long as there is no fault or change in the plant from nominal (reference model) conditions. On the other hand, any fault or change in the plant results in a nonzero residual vector. Recent surveys of the state of the art in such model based fault detection methods may be found in Patton et al. (1989), Gertler (1991), Frank $(1990,1991)$ and Patton and Chen (1991a).

One method of isolating a fault is by assigning directional properties to the residual vector through the design of observers with suitable dynamics. Beard (1971) and Jones (1973) first introduced this technique in the early 1970 s. In their method, which we will term BJDF (for Beard-Jones detection filter), the observer is designed such that the residuals generated by actuator faults propagate in a fixed direction in output space for any given fault. Extensions and applications of the BJDF have been reported, for example in Massoumnia (1986a,b), White and Speyer (1987), Massoumnia et al. (1989), Min (1987), Liubakka (1987), Rizzoni and Min (1991) and Park (1991).

The isolation of sensor faults to a fixed direction is not generally possible using the BJDF with the exception of sensors measuring uncontrolled inputs to the system. Faults in the latter sensors enter into the system equations in a

* Received 10 September 1992; revised 21 December 1992; revised 24 June 1993; received in final form 3 January 1994. This paper was not presented at any IFAC meeting. This paper was recommended for publication in revised form by Associate Editor Michèle Basseville under the direction of Editor Torsten Söderström. Corresponding author Professor Giorgio Rizzoni. Tel. +1 614292 2289; Fax +1 6142923163 .

$\dagger$ Department of Electrical Engineering and Computer Science, The University of Michigan, Ann Arbor, MI 48109, U.S.A.

$\ddagger$ Department of Mechanical Engineering, The Ohio State University, 206 W 18th Avenue, Columbus, OH 43210-1170, U.S.A. manner similar to actuator faults and are often termed pseudo-actuator faults. However, in the presence of faults in sensors measuring state variables the residual vector generated by the BJDF cannot be constrained to a fixed direction, but can only be constrained to lie in a plane. While this may still permit fault isolation, it would be desirable to extend the unidirectionality property of the BJDF to sensor faults.

This paper presents a method for representing any sensor faults in the form of an actuator fault, thereby permitting isolation of sensor faults to a fixed direction in the output space. The method is based on augmenting the state space to account for the dynamics of the sensor fault, and can be applied to both open-loop and closed-loop systems. This approach is mentioned but not developed by Massoumnia (1986a). The authors have recently been alerted to the fact that a similar result was also obtained by Massoumnia (1986b).

Several other approaches to sensor fault isolation using observer-based residual generators have been proposed in recent years. We mention, for example Min (1987), Caglayan (1980) and Emami-Naeini et al. (1988).

The problem of designing observers for linear systems with unknown inputs is one that has received much attention over the past several years. It has been shown by Viswanadham and Srichander (1987) and Frank (1990) that the unknown input observer (UIO) may be applied to the problem of detecting and isolating faults in linear systems. The UIO design as proposed by Viswanadham and Srichander (1987) and by Hou and Müller (1992) consists of transforming the system equations, such that the state vector can be divided into two parts-a part that can be directly obtained from the measurements, and another part consisting of the states that have to be estimated. A reduced-order observer can be designed to estimate these states, and the observer gains are selected so that they decouple the observer dynamics from the unknown input.

Another method of fault detection and isolation is to design observer-based residual generators that produce what are called structured residuals, i.e. residuals that exhibit certain special properties in the presence of particular faults. The most popular form of the structured residual is a residual that maintains a fixed direction in the parity space under the influence of a specific fault. Construction of observers using eigenstructure assignment lends itself naturally to the production of such unidirectional (under the influence of a fault) residuals. Patton et al. (1989) and Patton and Chen (1991a) demonstrate the use of eigenstructure assignment in FDI. Both left and right eigenvector assignment may be used to produce structured residuals that are robust to disturbances, and capable of isolating faults.

It is beyond the scope of this brief paper to discuss the very important issues of robustness to noise and model uncertainty and threshold selection [see for example Frank (1991) and Patton and Chen (1991b) for a discussion of these topics]. We will simply state that the design freedom that is available in the BJDF can be exploited to provide the desired properties. 
2. Dynamic system model

Consider the linear, time-invariant system

$$
\begin{gathered}
\frac{\mathrm{d} x(t)}{\mathrm{d} t}=A x(t)+B u(t), \\
y(t)=C x(t) .
\end{gathered}
$$

For simplicity, we shall omit the argument $(t)$, where $x \in \mathbf{R}^{n}$ is a state vector, $u \in \mathbf{R}^{p}$ is a control vector, $y \in \mathbf{R}^{m}$ is a measurement (or sensor) output vector, and $A, B, C$ are real matrices of compatible dimensions.

2.1. Actuator fault model. Assume that an actuator has failed in the system represented by equations (1) and (2). The fault can be modeled by an additional term in equation (1):

$$
\begin{gathered}
\frac{\mathrm{d} x}{\mathrm{~d} t}=A x+B u+\mathbf{f}_{i} n, \\
y=C x,
\end{gathered}
$$

where $f_{i} \in \mathbf{R}^{n}$ is defined as an actuator fault event vector (which is the column of B associated with the $i$ th actuator) and $n$ is a scalar function which represents the time evolution of the fault.

2.2. Sensor faults. Assume that a sensor failed in the system represented by equations (1) and (2). The fault can be modeled by an additional term added to equation (2):

$$
\begin{gathered}
\frac{\mathrm{d} x}{\mathrm{~d} t}=A x+B u, \\
y=C x+f_{\mathrm{s}} \zeta,
\end{gathered}
$$

where $f_{\mathbf{s}} \in \mathbf{R}^{m}$ is a sensor fault event vector and $\zeta$ is a scalar function which represents the evolution of the fault. Often, (but not always ) $f_{s}$ is a standard (unit) vector

$$
E_{i}=[0 \cdots 1 \cdots 0]^{\mathrm{T}},
$$

which represents the $i$ th sensor fault.

3. A new method for sensor fault detection and isolation In this section, a new method for detecting and isolating a sensor fault is formulated to overcome some of the limitations mentioned in the introduction. Given the system described by equations (1) and (2), we augment the state and output equations by one additional state. This auxiliary state describes the behavior of the sensor fault event which is to be diagnosed; the new output equation, however, does not contain the fault event vector, the fault event vector will appear as an additive term in the state equation and can therefore be treated as an actuator fault. The necessary and sufficient conditions that permit isolation of sensor faults to a fixed direction in the output space will also be given.

Consider a linear, time-invariant system with a sensor fault described by

$$
\begin{gathered}
\frac{\mathrm{d} x}{\mathrm{~d} t}=A x+B(u+K y), \\
y=C x+f_{s} \zeta(t),
\end{gathered}
$$

where $f_{s} \in \mathbf{R}^{m}$ is a sensor fault event vector, $\zeta$ is a scalar function which represents the evolution of the fault and $A$, $B, C$ are real matrices of compatible dimensions. Here, the output feedback term $K y$ is written separately since the output, $y$, is no longer free of fault. Note that this provision is made to account for both open-loop and closed-loop systems. Let us introduce the new system equations including the auxiliary state describing the dynamics of the sensor fault:

$$
\begin{gathered}
\frac{\mathrm{d} \bar{x}}{\mathrm{~d} t}=\bar{A} \bar{x}+\bar{B}\left(\begin{array}{c}
u+K y \\
\xi
\end{array}\right), \\
\bar{y}=\bar{C} \bar{x},
\end{gathered}
$$

where $\bar{x} \in \mathbf{R}^{n+1}$ is a new state vector, $\bar{A} \in \mathbf{R}^{(n+1) \times(n+1)}$ a new state matrix, $B \in \mathbf{R}^{(n+1) \times(p+1)}$ a new input matrix, $\bar{C} \in \mathbf{R}^{m \times(n+1)}$ a new output matrix and $\xi \in \mathbf{R}$ a sensor error input as defined below:

$$
\begin{gathered}
\bar{x}=\left[\begin{array}{l}
x \\
\zeta
\end{array}\right], \quad \bar{A}=\left[\begin{array}{cc}
A & 0 \\
0 & \alpha
\end{array}\right], \quad \bar{B}=\left[\begin{array}{ll}
B & 0 \\
0 & 1
\end{array}\right], \quad \bar{C}=\left[\begin{array}{ll}
C & f_{s}
\end{array}\right], \\
\xi=\frac{\mathrm{d} \zeta}{\mathrm{d} t}-\alpha \zeta,
\end{gathered}
$$

where 0 is a zero matrix of appropriate dimension. The differential equation for the auxiliary state variable, $\zeta$, given by

$$
\frac{\mathrm{d} \zeta}{\mathrm{d} t}=\alpha \zeta+\xi
$$

is always satisfied. This fact can be easily proved by substituting $\xi$ from equation (10) in equation (11). Therefore, the output $\bar{y}$ in equation (11) is always equal to the original output $y$. Note that $\alpha$ in (12) represents the additional degree-of-freedom in estimator design which is obtained by adding another state to the new state equations. It should be remarked that the fault signal $\zeta$ can be modeled by a linear system of arbitrary order, and that this fact may be exploited to best match the characteristics of the fault.

Observe, now, that equations (10) and (11) can be written

$$
\begin{gathered}
\frac{\mathrm{d} \bar{x}}{\mathrm{~d} t}=\bar{A} \bar{x}+\bar{B}\left(\begin{array}{c}
u+K y \\
0
\end{array}\right)+\left[\begin{array}{l}
0 \\
1
\end{array}\right] \xi, \\
y=\bar{C} \bar{x} .
\end{gathered}
$$

These are equivalent to the equations for a system with an actuator fault. For this system, a detection filter can be designed as follows:

$$
\begin{gathered}
\frac{\mathrm{d} \hat{x}}{\mathrm{~d} t}=\bar{A} \hat{x}+\bar{B}\left[\begin{array}{c}
u+K y \\
0
\end{array}\right]+D(y-\hat{y}), \\
\hat{y}=\bar{C} \hat{x}
\end{gathered}
$$

where $\hat{x}$ and $\hat{y}$ are the estimated state and estimated output, $u$ is the measured input, $y$ is the measured output and $D$ is the detection gain matrix. Note that equation (17) has measured output feedback $K y$ (not $K \hat{y}$ ).

By defining a state residual vector, $\bar{e}=\bar{x}-\hat{x}$, and an output residual vector, $q=y-\hat{y}$, we can write:

$$
\begin{gathered}
\frac{\mathrm{d} \bar{e}}{\mathrm{~d} t}=\frac{\mathrm{d} \bar{x}}{\mathrm{~d} t}-\frac{\mathrm{d} \hat{x}}{\mathrm{~d} t}=\bar{A}(\bar{x}-\hat{x})-D(y-\hat{y})+\bar{B}\left(\begin{array}{l}
0 \\
\xi
\end{array}\right) \\
=(\bar{A}-D \bar{C}) \bar{e}+\left(\begin{array}{l}
0 \\
1
\end{array}\right) \xi, \\
q=y-\hat{y}=\bar{C} \bar{e},
\end{gathered}
$$

Equation (19) has the same form as the error state equations with an actuator fault event vector, $[0,1]^{\mathrm{T}}$. Hence, any BJDF design algorithm can be used to determine the gain matrix $D$.

It is a well-known result that the necessary and sufficient condition for detectability of the additive fault event vector $[0,1]^{\mathrm{T}}$ is that the fault event vector is detectable if and only if $(A, C)$ is observable (Beard, 1971). In addition, we will now prove that the same requirement may be expressed in terms of the original system and output matrices, $A$ and $C$. Lemmas 3.1 and 3.2 summarize this new formulation of sensor fault detection, by giving a necessary and sufficient condition for sensor fault detectability.

Lemma 3.1. Let $v_{1}, v_{2}, \ldots, v_{n}$ be eigenvectors of $A$. $(A, C)$ is observable iff

$$
C v_{i} \neq 0 \quad \forall i=1,2, \ldots, n .
$$

For a proof, see Park (1991). The following lemma guarantees the necessary and sufficient condition:

Lemma 3.2. $(\bar{A}, \bar{C})$ is observable iff $(A, C)$ is observable. 
Proof. Let $v_{1}, v_{2}, \ldots, v_{n}$ and $w_{1}, w_{2}, \ldots, w_{n}$ be eigenvectors of $A$ and $\bar{A}$, respectively. From the definition of $\bar{A}$

$$
\vec{A}=\left[\begin{array}{ll}
A & 0 \\
0 & \alpha
\end{array}\right]
$$

it is easy to see that eigenvectors of $\bar{A}$ have the following forms:

$$
w_{1}=\left[\begin{array}{c}
v_{1} \\
0
\end{array}\right], \quad w_{2}=\left[\begin{array}{c}
v_{2} \\
0
\end{array}\right], \ldots, w_{n}=\left[\begin{array}{c}
v_{n} \\
0
\end{array}\right], \quad w_{n+1}=\left[\begin{array}{l}
0 \\
1
\end{array}\right] .
$$

By Lemma 3.1, the pair $(\bar{A}, \bar{C})$ is observable iff the output matrix $\bar{C}$ and the eigenvectors satisfy the following condition:

$$
\bar{C} w_{i} \neq 0 \quad \forall i=1,2, \ldots, n+1 .
$$

But

$$
\bar{C} w_{i}=\left[\begin{array}{ll}
C & f_{\mathrm{s}}
\end{array}\right] w_{i}=\left\{\begin{array}{ll}
C v_{i} & i=1,2, \ldots, n \\
f_{\mathrm{s}} & i=n+1
\end{array} .\right.
$$

From equation (25), we see that

$$
\bar{C} w_{i} \neq 0 \text { iff } C v_{i} \neq 0 .
$$

Therefore, by Lemma 3.1 and equation $(26),(\bar{A}, \bar{C})$ is observable. Finally, the following theorem gives the necessary and sufficient condition for sensor fault detectability for the method proposed in this paper:

Theorem 4.1. Consider the system of equations (8) and (9). The sensor fault, $f_{\mathrm{s}}$, is detectable and can be isolated (by constraining output error residual in one-dimensional subspace by choice of $D$, i.e. in the sense of the $B J D F$ ) iff $(A, C)$ is observable.

\section{Proof. Apply Lemma 3.2 to equations (24) and (25).}

The advantage of this sensor fault detection method over previous methods is simply that it converts the sensor fault problem to an actuator fault problem. This method is applicable to sensor fault detection in both open- and closed-loop systems.

\section{Conclusion}

This paper has presented an extension of the well-known Beard-Jones detection filter that permits the isolation of sensor faults in a dynamic system to a fixed direction. The method is based on augmenting the system equations by an auxiliary state to represent the dynamic behavior of the sensor fault, and in effect converts the sensor fault into the same form as an actuator fault. The only condition required is observability of the original system. This method can be applied to both open-loop and closed-loop systems.

\section{References}

Beard, R. V. (1971). Fault accommodation in linear systems through self-reorganization. Report MVT-71-1, ManVehicle Laboratory, Massachusetts Institute of Technology, Cambridge, MA.

Caglayan, A. (1980). Necessary and sufficient conditions for detectability of jumps in linear systems. IEEE Trans. on Autom. Control, AC-25, 833-834.

Emami-Naeini, A., M. M. Akhter and S. M. Rock (1988). Effect of model uncertainty on failure detection: the threshold selector. IEEE Trans. on Autom. Control, AC-33, 1106-1115.

Frank, P. M. (1990). Fault detection in dynamic systems using analytical and knowledge-based redundancy-a survey and some new results. Automatica, 26, 459-474.

Frank, P. M. (1991). Enhancement of robustness in observer-based fault detection. In Proc. IFAC, IMACS Safeprocess Symposium, Baden-Baden, Germany.

Gertler, J. (1991). A survey of fault detection and identification methods. In Preprints of IFAC, IMACS Safeprocess Symposium, Baden-Baden, Germany.

Hou, M. and P. C. Müller (1992). Design of observers for linear systems with unknown inputs. IEEE Trans. on Autom. Control, 37, 871-875.

Jones, H. L. (1973). Fault detection in linear systems. Report T-608, The Charles Stark Draper Laboratory, Cambridge, MA.

Liubakka, M. (1987). Application of fault detection and isolation theory to internal combustion engines. M.S. Thesis, EECS Department, University of Michigan, Ann Arbor, MI

Massoumnia, M. A. (1986a). A geometric approach to failure detection and identification in linear systems. Ph.D. Thesis, MIT, Department of Aeronautics and Astronautics, Cambridge, MA.

Massoumnia, M. A. (1986b). A geometric approach to the synthesis of fault detection filters. IEEE Trans. on Autom. Control, AC-31, 839-846.

Massoumnia, M. A., G. C. Verghese and A. S. Willsky (1989). Failure detection and identification. IEEE Trans. on Autom. Control, AC-34, 316-321.

Min, P. S. (1987). Detection of incipient failures in dynamic systems. Ph.D. Thesis, EECS Department, University of Michigan, Ann Arbor, MI.

Park, J. (1991). A unified theory of fault detection filters. Ph.D. Thesis, EECS Department, University of Michigan, Ann Arbor, MI.

Patton, R. J. and C. Chen (1991a). Model based fault detection and identification. In Preprints of IFAC, IMACS Safeprocess Symposium, Baden-Baden, Germany.

Patton, R. J. and C. Chen (1991b). Robust fault detection using eigenstructure assignment: a tutorial consideration and some new results. In Proc. of the 30th Conf. on Decision and Control, Brighton, England, pp. 2242-2247.

Patton, R., R. Patton, P. Frank and R. Clark (1989). Fault Diagnosis in Dynamic Systems, Theory and Applications, Prentice-Hall, Englewood Cliffs, NJ.

Rizzoni, G. and P. S. Min (1991). Detection of sensor faults in automotive engines. IEEE Trans. on Vehicular Technology, 40, 487-500.

White, J. E. and J. L. Speyer (1987). Detection filter design: spectral theory and algorithms. IEEE Trans. on Autom. Control, AC-32, 593-603.

Viswanadham, N. and R. Srichander (1987). Fault detection using unknown input observers. Control-Theory and Advanced Technology, 3, 91-101. 